\title{
A DERIVATIVE CONTROL MECHANISM FOR SUPPLY CHAIN PERFORMANCE IMPROVEMENT
}

\author{
Olatunde Temitope Baruwa \\ Miquel Àngel Piera \\ Department of Telecommunications and System Engineering (LogiSim) \\ Universitat Autònoma de Barcelona, Barcelona, Spain. \\ Email: OlatundeTemitope.Baruwa@campus.uab.cat, MiquelAngel.Piera@uab.es
}

\section{KEYWORDS}

Derivative Control, Simulation, Coloured Petri Net, Supply Chain.

\begin{abstract}
Organizations increasingly find that they must rely on effective supply chains to successfully compete in the global market and networked economy. Over the years, simulation models have proved to be a useful decision support tool for examining the performance of different system configurations and/or alternative operating procedures for complex systems to improve operational and organizational efficiency. This paper presents a new approach that transforms an optimization problem to a search problem by evaluating the interrelationship of decision making when either one or more actors in the supply chain take aggressive or conservative positions. This is aimed at improving and optimizing performance, both individually and for the entire chain in order to reduce costs, lower inventories, and increase manufacturing throughput.
\end{abstract}

\section{INTRODUCTION}

The complexity of supply chain systems requires the design of new decision support systems (DSS) that can evaluate and analyze the effects of an action applied to any of the sub-systems. Such a DSS requires a new modelling approach with a deeper understanding of the nature of the complexity itself. The effect of an action is propagated through the chain in different ways according to the interrelationship of independent components. Thus, a DSS must be able to accommodate perturbations that might occur and propagate at any point within the system.

Modern-day technology has become a vital tool in making important business decisions with a growing number of commercial packages, Advanced Planning and Scheduling applications and Enterprise Resource Planning (ERP) solutions employed to optimize supply chains. From the research community, some new developments such as connectionist approaches developed by the artificial intelligence community have provided different methodologies for optimizing practice based knowledge, just by codifying the expert experience in form of case histories [3]. Nevertheless, most of these approaches are unable to give explanations about how optimal solutions are reached. Over the years, simulation models have proved to be a useful decision support tool for examining the performance of different system configurations and/or alternative operating procedures for complex systems to improve operational and organizational efficiency. The model must represent in the most simplified way, the dynamic interests of the real system which should allow understanding, describing, modifying and possibly controlling the behaviour of the system.

The model developed in this paper is a classical supply chain business model based on the beer game ideology developed at the Sloan School of Management in early 1960's [17] where the goal of the participants is to minimize costs either individually or globally. It is modelled as a discrete-event system with four stages having one participant at each stage. Customer satisfaction through the most efficient use of resources while minimizing costs serves as the primary objective of the supply chain. The business model considers order processing, fulfilment and delivery of orders, decisions on the quantity to produce or order and the effect of these decisions on the supply chain in order to maximize customer service level.

Several simulation modelling approaches to tackle supply chain decision problems, optimizing and improving the supply chain performance have been proposed. David Simchi-Levi et al(Beer Game Authors) developed a Web/Java based model that seek to clarify the advantages of taking an integrated approach to managing the supply chain; the value of sharing information across the supply chain components by demonstrating the bullwhip effect. [3] developed a timed-hierarchical Coloured Petri Net for the simulation and performance of the beer game supply chain model to study the bullwhip effect. [10] extended the simulation model developed by [3] using different ordering policies to demonstrate the bullwhip effect and analyse the impact of standard demand forecasting methods on the performance of the system.

A four parameter discrete model developed by [14] and the use of genetic algorithm techniques was proposed in [15] to simulate the effects of two ordering policies under a particular customer demand to optimize the performance of the chain. This approach restricts some parameters that might be of great interest to 
improve the performance of the supply chain. In [1], [6] and [7], a Just-in-Time Petri Net model, Artificial agent based model and Arena software based supply chain model respectively were proposed to complement and understand the complexity of managing decision making in the beer distribution supply chain game.

In general, the developed models demonstrate and validate the bullwhip effect observed in supply chains and they are able to provide good solutions under standard conditions. However, none of these approaches can explain or justify why the optimal solution is obtained which would be very useful to deal with optimal or quasi-optimal solutions when the system is operating under different context conditions. When the dynamics of the problem changes, it is almost impossible to obtain a near optimal solution since the agents that drive the solution are unknown. Moreso, these models are not useful to understand the causeeffect relationship when either one or more of the actors in the supply chain takes an aggressive or conservative position. This is critical to the performance of any supply chain decision support system.

In order to solve this problem, the model should help to understand the consequences of a decision at a certain level. To deal with this, the model should give a proper solution to the following questions:

- Which are the consequences of firing, delaying or disabling a certain activity? And how are such actions propagated in the supply chain?

- Does the model support new dynamics specification?

- Does the model permit automatic evaluation of different policies using the same model?

- Does the model support the combination of simulation for global optimization and decision making in complex operations?

- Does the model provide feasible, quasi-optimal and optimal solutions in a timely fashion?

This paper presents a new approach that transforms an optimization problem to a search problem by evaluating the interrelationship of decision making when either one or more actors in the supply chain takes aggressive or conservative positions and fulfilling the above mentioned characteristics.

The paper is structured as follows: The next section describes the supply chain model and the major activities considered in the model. The third section discusses modelling formalisms while the fourth section gives the description of the formalism employed and the developed model. The fifth section focuses on the proposed approach and the results obtained are presented in the sixth section. Finally, the last section gives a summary of the paper and ideas for future research.

\section{THE BUSINESS (SUPPLY CHAIN) MODEL}

It is worth mentioning that there is a clear distinction between Business models and Production models. A business model is not a description of a complex social system itself with all its actors, relations and processes. Rather, it describes the logic of a 'business system' for creating value that lies behind the actual processes [11]. [9] defines business model as the managerial equivalent of the scientific method - you start with a hypothesis, which you then test in action and revise when necessary. Nevertheless, a business model should not be misconstrued as a strategy. A strategy is what makes you unique in the market place. However, performance of real production systems is very sensitive to various kinds of factors such as unexpected delays, queuing, breakdowns, operation time and inadequate labour on a day to day basis. Thus, a business model should also incorporate these low abstraction level aspects (i.e. production model)

Effective inventory management strategies and their efficient implementation are recognized as important elements in a business model. The targets are reduction of the level and value of inventory maintained, high speed in transforming them to orders delivered to customers and flexibility in dealing with changing customer demand[5]. We consider a decentralized four stage supply chain consisting of a Manufacturer, Distributor, Supplier and Retailer based on the Beer Distribution Game developed to demonstrate a number of key principles of supply chain management. We assume that there is a single participant at each stage. Figure 1 highlights the major activities carried out by each participant in the supply chain. In the decentralized supply chain, each participant possesses good local information (about his inventory, remaining orders, receiving amounts from his upstream supplier and amounts delivered to the downstream participant), but not in possession of global information.

A supply chain must be able to handle a significant amount of events, both expected and unexpected. The unexpected events, also called exceptions or perturbations, typically arise because there is usually a gap between supply chain planning and execution [8]. As described in figure 1, the main events to be considered are: order filling, fulfilment of orders, delivery and the decision on the quantity to order or produce. The decision made on the quantity to order or produce plays a significant role in evaluating the overall effective performance of the supply chain. When decisions on what to order comes to play, there exists two possibilities; the possibility of having excess stock with low demand or the possibility of excess demand with low stock. The presented approach tends to strike a balance between the two so that the expected demand is closer to the actual demand in order to avoid out of stock and unnecessary back orders. 


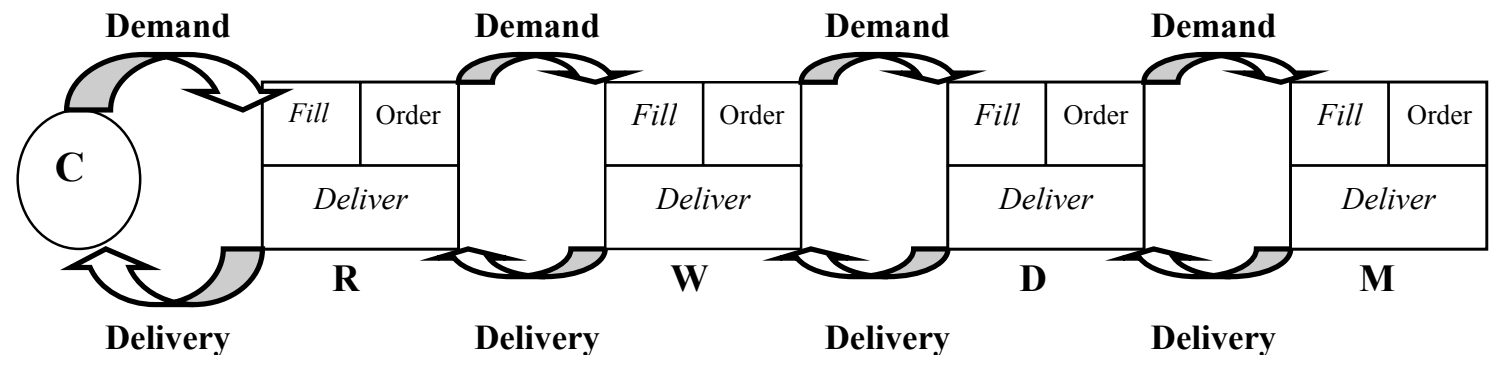

Figure 1: The Supply Chain Model

Key: C-Consumer; R-Retailer; W-Wholesaler; D-Distributor; M-Manufacturer

The model considers a "Derivative" control mechanism for managing these events and responding to them dynamically when perturbations occur. These events can be implemented if the modeller perceives that each action such as placing an order, delivering a product, or taking a decision regarding inventory levels is a discrete event with dynamic properties [10]. Since the number of reachable states in a supply chain is typically very large it is necessary to describe clearly all activities associated with events.

The triggering of an event can give rise to several consequences. For example, if an order is placed on a participant in a particular period and there is no sufficient inventory to fill the order, this results to a back $\log$ of order, a possible out of stock in the next period and a possible aggressive order placement on the upstream participant. Each time an order is placed on a participant, it is first checked whether the amount of products in inventory is sufficient to fill it. In case the amount is sufficient, the order placed will be delivered to the downstream component. Otherwise, this generates back order that will be fulfilled along with a new order placed in the next period. The supply chain model allows the delivery of incomplete orders. When an order is received, it is checked against the order placed. If an incomplete order is delivered, this might trigger an unwanted demand in the next period by the downstream participant depending on the pattern of orders it receives.

\section{MODELLING FORMALISM}

A great deal of formalisms for modelling discrete event systems exists in the literature. Amongst them are: Flow Charts, Block Diagrams, Automata, Temporal Logic, Calculus of Events, Artificial Intelligence, Discrete Event System Specification(DEVS) and Classical Petri Nets(PN). Business models require a formalism that is dynamic, descriptive, depicts causality, easily accessible interpretation and able to integrate every aspect of the system specification. Most of these formalisms do not support the specification of low level production aspects which are necessary to implement the proposed decision making approach. Though, classical PN provides an appealing graphical representation which facilitates easy understanding of the system behaviour and the synchronisation of concurrent processes, it does not support information flow for decision making which is essential for any DSS.

The evolution of Coloured Petri Nets (CPN) has been driven by the desire to develop a modelling language at the same time theoretically well-founded and versatile enough to be used in practice for systems of the size and complexity that can be found in typical industrial projects [4]. CPN supports specification, information flow and the manipulation of data values through a functional programming language. The main advantages of CPN models are given in [4]. The main CPN components that fulfil the cause-effect event relationship analysis include: places, transitions, input arc expressions, colour sets and state vector. See [12, 15] for the description of these terms and tutorial on CPN.

\section{THE CPN MODEL}

The proposed approach is implemented on CPN Tools software developed and maintained by the CPN Group, University of Aarhus, Denmark [16]. CPN allows the representation of a system in a composite structure with few places and transitions. To analyze and understand the cause-effect relationship of the system in an efficient way the model elements have been represented at the same level rather than the hierarchical structures developed by other authors to simply serve simulation purposes.

The supply chain is modelled as a timed net with the global clock of the CPN tool used as the weekly timer (time 1 represents week 1 , time 2 represents week 2 etc). Each time the global clock increments, this implies that the cycle of events for a particular week has been completed. Within each week, all the possible events in the supply chain must be completed before the clock changes. Figure 2 shows the CPN supply chain model and tables 1, 2, 3 and 4 summarize the meaning of the place nodes, transitions (events), colours and functions. It should be noted that the same model can be used when events behave as a random variable.

The system is described by 9 transitions classified in a group of 4 major activities - order filling (T1, T2), delivery (T5, T6, T7, T8), decision on quantity to order 


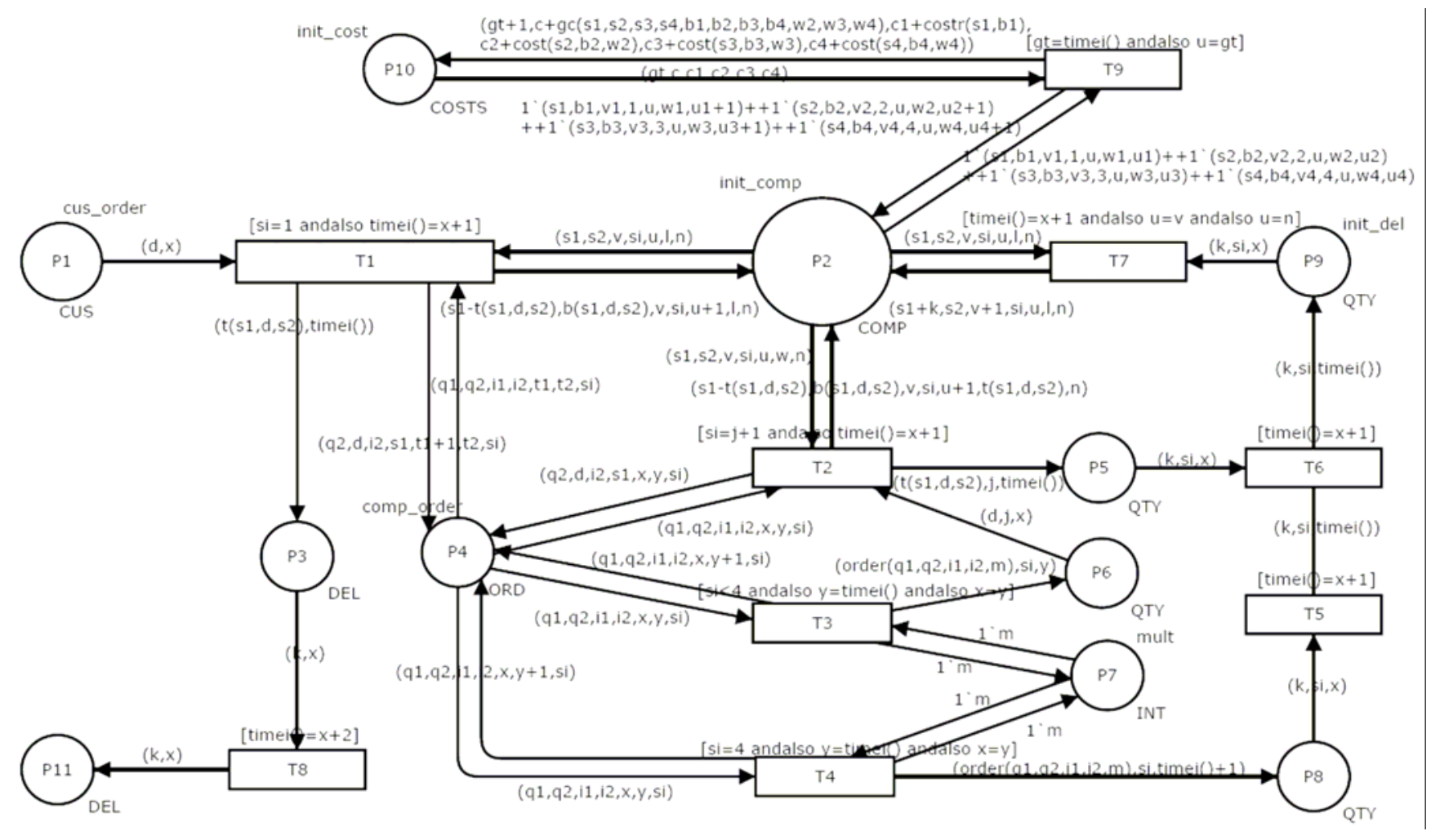

Figure 2: The CPN Model of the Supply Chain

Table 1: Event Description

\begin{tabular}{|c|l|}
\hline Transition & \multicolumn{1}{|c|}{ Description } \\
\hline T1 & Filling of customer orders by the retailer and updating P4 for the computation of new orders. \\
\hline T2 & $\begin{array}{l}\text { Filling of weekly retailer, wholesaler and distributor orders by the wholesaler, distributor and } \\
\text { manufacturer respectively and updating P4 for the computation of new orders. }\end{array}$ \\
\hline T3 & Computation of weekly orders by retailer, wholesaler and distributor. \\
\hline T4 & Computation of quantity to produce by manufacturer. \\
\hline T5,T6 & One week delay for quantity produced and filled orders. \\
\hline T7 & Delivery of orders after the two-week delay to be added to inventory. \\
\hline T8 & Delivery of customer orders after the two-week delay. \\
\hline T9 & Computation of weekly global cost and individual component cost. \\
\hline
\end{tabular}

Table 2: Place Nodes Information

\begin{tabular}{|c|l|l|}
\hline Place & Colour & \multicolumn{1}{|c|}{ Description } \\
\hline P1 & $(\mathrm{d}, \mathrm{x})$ & Weekly customer orders \\
\hline P2 & $(\mathrm{s} 1, \mathrm{~s} 2, \mathrm{v}, \mathrm{si}, \mathrm{u}, \mathrm{l}, \mathrm{n})$ & $\begin{array}{l}\text { Component Information(inventory, back order, control for delivery, } \\
\text { component identifier, order filling control, delivered orders, cost } \\
\text { control) }\end{array}$ \\
\hline P3 & $(\mathrm{k}, \mathrm{x})$ & Quantity to deliver to customer \\
\hline P4 & $(\mathrm{q} 1, \mathrm{q} 2, \mathrm{i} 1, \mathrm{i} 2, \mathrm{x}, \mathrm{y})$ & $\begin{array}{l}\text { History of previous and current quantity demanded and inventory } \\
\text { level. }\end{array}$ \\
\hline P5,P8,P9 & $(\mathrm{k}, \mathrm{si}, \mathrm{x})$ & Quantity to deliver \\
\hline P6 & $(\mathrm{d}, \mathrm{j}, \mathrm{x})$ & Quantity to order by retailer, wholesaler and distributor. \\
\hline P7 & $\mathrm{m}$ & Multiplier \\
\hline P10 & $(\mathrm{gt}, \mathrm{c}, \mathrm{c} 1, \mathrm{c} 2, \mathrm{c} 3, \mathrm{c} 4)$ & Cost (global, retailer, wholesaler, distributor, manufacturer) \\
\hline P11 & $(\mathrm{k}, \mathrm{x})$ & Customer delivered orders \\
\hline
\end{tabular}


Table 3: Function Description

\begin{tabular}{|c|c|}
\hline Function/Value & Description \\
\hline cus order & Weekly customer orders \\
\hline comp_order & $\begin{array}{l}\text { Initial marking for order computation } \\
1^{`}(4,4,4,4,0,0,1)++1^{`}(4,4,4,4,0,0,2)++1^{`}(4,4,4,4,0,0,3)++1^{`}(4,4,4,4,0,0,4)\end{array}$ \\
\hline init_del & 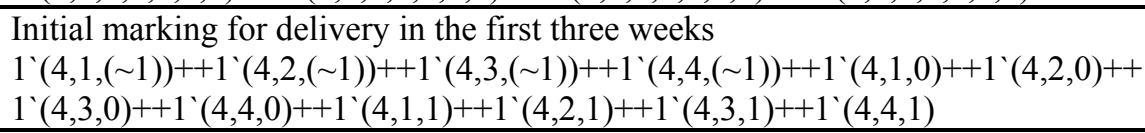 \\
\hline mult & Marking for multiplier $\left(1^{\prime}(1)++1^{`}(2)++1^{`}(3)++1^{\prime}(4)\right)$ \\
\hline init_comp & $\begin{array}{l}\text { Initial marking for component information } \\
1^{`}(0,0,0,1,0,0,0)++1^{`}(0,0,0,2,0,0,0)++1^{`}(0,0,0,3,0,0,0)++1^{\prime}(0,0,0,4,0,0,0)\end{array}$ \\
\hline init_cost & Initial cost $(1(1,36,0,12,12,12))$ \\
\hline $\mathrm{t}(\mathrm{s} 1, \mathrm{~b}, \mathrm{~s} 2)$ & Computation of quantity to be delivered \\
\hline $\mathrm{b}(\mathrm{s} 1, \mathrm{~b}, \mathrm{~s} 2)$ & Back order computation \\
\hline $\operatorname{order}(\mathrm{q} 1, \mathrm{q} 2, \mathrm{i} 1, \mathrm{i} 2, \mathrm{~m})$ & Computation of quantity to order/produce by each component \\
\hline $\mathrm{gc}(\mathrm{s} 1, \mathrm{~s} 2, \mathrm{~s} 3, \mathrm{~s} 4, \mathrm{~b} 1, \mathrm{~b} 2, \mathrm{~b} 3, \mathrm{~b} 4)$ & Global cost computation \\
\hline $\operatorname{cost}(\mathrm{s} 1-4, \mathrm{~b} 1-4, \mathrm{w} 1-\mathrm{w} 4)$ & Component cost computation \\
\hline timei() & Global clock \\
\hline
\end{tabular}

or produce (T3, T4) and cost computation (T9). The function "order ( )" in the model shows the "derivative" control mechanism implemented in the supply chain for decision making. This will be fully discussed in the next section. An initial steady state is assumed in the supply chain. During the first three weeks, the customer and the participants order constant demands per week, the supply chain members hold the same quantity in inventory as the customer's demand in the first week and the same quantity is delivered to the supply chain members in the subsequent two weeks. The participants can order as much as they want in the beginning of the fourth week. The transition guard expressions in the model ensure the synchronization, sequence and priority of events with the use of the colours embedded in each place. Place P2 represents the participants - retailer, wholesaler, distributor and manufacturer with each having an identifier. This can be modelled with more participants at each stage as CPN allows the inclusion of more parameters with the use of colours.

\section{PROPOSED APPROACH - DERIVATIVE CONTROL MECHANISM}

Simulation gives different solutions according to each scenario. With this, a manager can only understand how the system works; he has no information on how to improve the reality. The derivative control mechanism introduced in the model explores all possible existing solutions and a decision maker is able to understand the effect of each decision taken at any point in time on his own system and the entire chain as a whole. The approach also incorporates the flexibility in dealing with changing customer demands.

To enhance the formal analysis of the model, the control mechanism is supported with the application of the coverability tree (CT). It is generally believed that adding more parameters to the system in one single model makes the size of the state space grows exponentially. However, the use of the coverability tree coupled with heuristics tackles the state space explosion [13] by running a check on every path of the tree to identify dead-end nodes, stop the possible expansion of old nodes (same markings) and the successive increments of markings on each node for all reachable states. Table 5 shows the logic table for the derivative control mechanism (function "order ( )" in the model).

The decision mechanism is driven by changes in demand and inventory levels whose derivative is taken over two (2) periods ( $\mathrm{n}$ and n-1). This is weekly ordering or production decision in an effort to satisfy downstream demands. A multiplier " $\mathrm{m}$ " with values ranging from 1 to 4 is introduced in order to evaluate the conservative and aggressive positions of the actors involved in the chain. Let's consider decisions 1,4 , and 8 as an example:

Decision 1: Order or produce the current demand when there is no change in demand and inventory level.

Decision 4: Order or produce the multiplier of the current demand if there is an increase in changes in quantity demanded and no inventory level change.

Decision 8: A decision to order or produce the divisor of the current demand implies a decrease in quantity demanded and an incremental change in inventory level.

As a result, it allows checking a variety of state space solutions, opening the whole tree, understanding the cause-effect relationship of each action and how the actions are propagated through upstream and downstream. Since the model developed is not just for simulation but a decision support system that would allow the stakeholder to choose from a variety of solutions the model presents. In essence, the approach transforms the optimization problem to a search problem in which a decision maker can select a near optimal solution that best fits the current situation in a 
Table 4: Derivative Control Mechanism

\begin{tabular}{|c|c|c|c|}
\hline & $\nabla \mathbf{D n}$ & $\nabla \mathbf{I n}$ & Decision \\
\hline 1 & 0 & 0 & $\mathrm{Dn}$ \\
\hline 2 & 0 & 1 & $1 / \mathrm{m} * \mathrm{Dn}$ \\
\hline 3 & 0 & -1 & $\mathrm{~m} * \mathrm{Dn}$ \\
\hline 4 & 1 & 0 & $\mathrm{~m} * \mathrm{Dn}-\mathrm{i}$ \\
\hline 5 & 1 & 1 & Dn-i \\
\hline 6 & 1 & -1 & $\mathrm{~m} * \mathrm{Dn}$ \\
\hline 7 & -1 & 0 & Dn-i \\
\hline 8 & -1 & 1 & $1 / \mathrm{m} * \mathrm{Dn}-\mathrm{i}$ \\
\hline 9 & -1 & -1 & $\mathrm{Dn}$ \\
\hline Key & \multicolumn{2}{|c|}{$-1-$ Decrease } & 1 - Increase \\
\hline \multicolumn{4}{|c|}{$\nabla \mathrm{Dn}=(\mathrm{Dn}-\mathrm{Dn}-\mathrm{i}) / \nabla_{\mathrm{n}}$} \\
\hline \multicolumn{3}{|c|}{$\mathrm{Dn} / \mathrm{In}$ - Demand/Inventory at week n } & Dn-i/ In-i - Demand/Inventory at week (n-1) \\
\hline \multicolumn{3}{|c|}{$\mathrm{m}$ - Multiplier $(1,2,3,4) ; \mathrm{i}=1$} & $\forall \mathrm{n}=1,2 \ldots \mathrm{N}(\mathrm{N}$ is the total number of weeks) \\
\hline
\end{tabular}

timely fashion. The next section justifies the approach employed with the results obtained.

\section{RESULTS}

The model was driven by a step change in customer demand as shown in Figure 3 for a period of 15 weeks. Given the change in demand, the system can be ruled according to different costs. Figure 4 shows the global costs obtained for the entire chain during this period. The state space generated a good number of solutions at the end of this period in which 190 were collected for analysis. The system cost range from $\$ 524$ to $\$ 1052$. The inventory holding cost attracts a charge back of $\$ 1$ weekly per case while a penalty of $\$ 2$ for back orders.

Most of the policies will lead the system to high production cost and huge penalties. However, with state space analysis, it is possible to obtain a policy that supports customer demand at a very minimal cost $(\$ 524$ - marked point on the graph). The policy that drives the system to the optimal cost during the first ten weeks is shown in Figure 5. The figure gives a clearer view of how customer demands are propagated from downstream to upstream. Participants take positions based on downstream demand and periodical inventory levels. Also, a steady state was maintained by the participants during the first six weeks. After the fourth week, the position of the retailer changed due to upward change in customer demand in the fourth week. The retailer's reaction to this change prompted the wholesaler to re-adjust his position on the chain. This affected the next two upstream participants in the subsequent weeks.

\section{CONCLUSION}

A derivative control mechanism for supply chain performance optimization has been proposed. The approach implemented supports the integration of simulation for global optimization and decision making. The efficiency of the approach was shown in the results section.

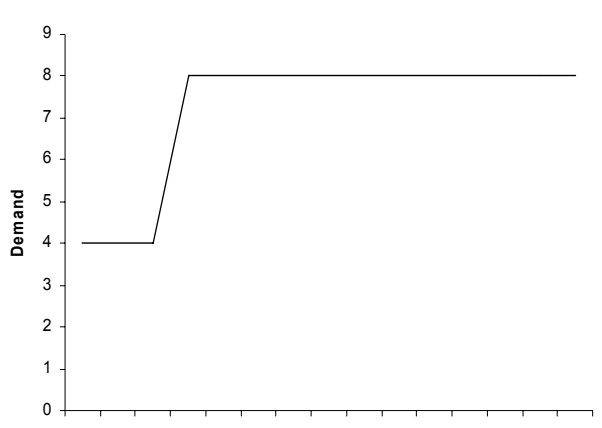

Figure 3: Customer Demand

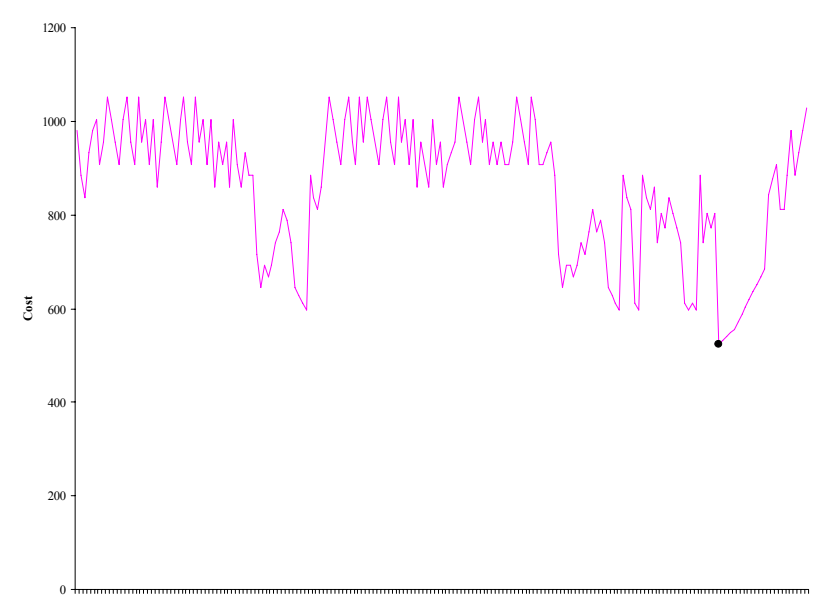

Figure 4: Global Cost

In order to reduce computational time and the size of the coverability tree, future research work should focus on the introduction of heuristics to prune the search space. Also, the effects of taken the derivative over a longer period can be considered with respect to product lead time. 


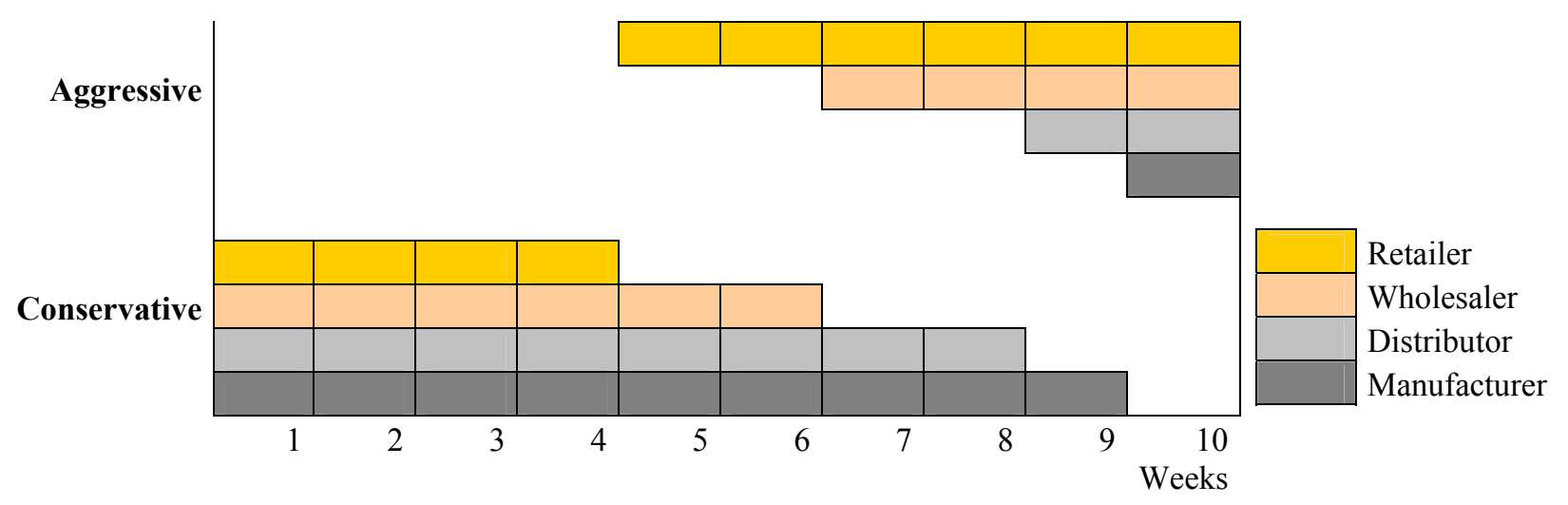

Figure 5: Positions of Participants for the Optimal Policy

\section{REFERENCES}

[1] Desrochers A. and Pia Fanti M. 2005, "A Supply Chain Model Using Complex-valued Petri Nets" Proceedings of the IEEE International Conference on Robotics and Automation.

[2] Dias W.P.S 2007, "Philosophical Grounding and Computational Formalization for Practice Based Engineering Knowledge" Knowledge Based Systems 20.

[3] Dragana M. et al 2004, "Bullwhip Effect And Supply Chain Modelling And Analysis Using CPN Tools", Proc. of the 5th Workshop and Tutorial on Practical Use of Coloured Petri Nets and the CPN Tools. Aarhus, Denmark 219-234.

[4] K. Jensen 1997, "Coloured Petri Nets. Basic Concepts, Analysis Methods and Practical Use" Volume 1, Basic Concepts. Monographs in Theoretical Computer Science, Springer-Verlag, 2nd corrected printing 1997. ISBN: 3-540-60943-1.

[5] Ka-Fu C. and Chi-Fai C. 2006, "A Manufacturing Supply Chain Business Model and Its Implementation in a Plastics Manufacturing Company" Materials Science Forum Vols. 532-533 Pg 1096-1099.

[6] Kimbrough S.O. et al 2001, "Computers Play the Game: Can Artificial Agents Manage Supply Chains? 34th Hawaii International Conference on System Sciences.

[7] Kumar S. et al 2007, "Demonstrating Supply Chain Parameter Optimization Through Beer Game Simulation, Information" Knowledge, Systems Management, Volume 6, Number 4 Pg 291-322.

[8] Liu R. et al 2007, "A Formal Modelling Approach for Supply Chain Event Management" Decision Support Systems, Volume 43, Issue 3, Pg $761-778$.

[9] Magretta J. 2001, "Why Business Models Matter" Harvard Business Review Article, May PN R0205F

[10] Papanagnou C. I. and Halikias G. D. 2006, "Analysing Different Ordering Policies in a Series Supply Chain by Using Coloured Petri Nets" 20th European Conference on Modelling and Simulation, ECMS.

[11] Petrovic, O. et al 2001, "Developing Business Models for eBusiness" Proceedings of the International Conference on Electronic Commerce,, Vienna, Austria, October 31 -- November 4.

[12] Piera M.A et al 2004, "Optimization of Logistic and Manufacturing Systems through Simulation: A Colored Petri Net-Based Methodology". Simulation Journal, V. 80 (1). 2004.
[13] Sònia B. et al 2005, "Specification of Metaheuristics in Colored Petri Net Models"International Mediterranean Modelling Multiconference, EMSS

[14] Sterman J.D 1989, "Modelling Managerial Behaviour: Misperceptions of Feedback in A Dynamic Decision Making Experiments" Management Science $35 \mathrm{Pg}$. 321-379.

[15] Strozzi F. et al 2006, "Beer Game Order Policy Optimization under Changing System Demand" Science Direct (Elsevier BN) Pg. 2153-2163.

[16] http://wiki.daimi.au.dk/cpntools/cpntools.wiki

[17] http://beergame.mit.edu/

\section{AUTHOR BIOGRAPHIES}

OLATUNDE T. BARUWA holds a bachelor's degree in Electronic and Computer Engineering (Lagos State University, Nigeria, 2004) and a master's degree in Management (Universitat Pompeu Fabra (UPF), Spain, 2007). He is currently a PhD student in the Department of Telecommunication and Systems Engineering at the Universitat Autònoma de Barcelona, Spain where he is developing his dissertation and a member of LogiSim, a Modelling and Simulation Institution sponsored and founded by the local government of Catalunya. Prior to studying at UPF, he worked at the Lagos Business School, Nigeria for 2 years. His research interests are in the areas of modelling, simulation and optimization of logistic systems, industrial processes and supply chain management. His e-mail address is olatundetemitope.baruwa@uab.cat.

MIQUEL ÀNGEL PIERA I EROLES (DR.) received his MSc (Control Engineering) from the University of Manchester Institute of Technology in 1990 and his $\mathrm{PhD}$ degree from the Autonomous University of Barcelona (Spain) in 1994. He participates in industrial research projects in the logistics and manufacturing field and at present he is Co-director of LogiSim, a Modelling and Simulation Institution sponsored and founded by the local government of Catalunya. Professor Piera has been the coordinator of the Spanish Simulation group of the International Federation of Automatic Control. Recently, he has published a modelling and simulation book that is being used for teaching in many Spanish Universities. 